\title{
Systematic engineering of the central metabolism in Escherichia coli for effective production of $n$-butanol
}

\author{
Mukesh Saini', Si-Yu Li ${ }^{2}$ Ze Win Wang ${ }^{1}$, Chung-Jen Chiang ${ }^{3^{*}}$ and Yun-Peng Chao ${ }^{1,4,5^{*}}$
}

\begin{abstract}
Background: Microbes have been extensively explored for production of environment-friendly fuels and chemicals. The microbial fermentation pathways leading to these commodities usually involve many redox reactions. This makes the fermentative production of highly reduced products challenging, because there is a limited NADH output from glucose catabolism. Microbial production of $n$-butanol apparently represents one typical example.

Results: In this study, we addressed the issue by adjustment of the intracellular redox state in Escherichia coli. This was initiated with strain BuT-8 which carries the clostridial CoA-dependent synthetic pathway. Three metabolite nodes in the central metabolism of the strain were targeted for engineering. First, the pyruvate node was manipulated by enhancement of pyruvate decarboxylation in the oxidative pathway. Subsequently, the pentose phosphate (PP) pathway was amplified at the glucose-6-phosphate (G6P) node. The pathway for G6P isomerization was further blocked to force the glycolytic flux through the PP pathway. It resulted in a growth defect, and the cell growth was later recovered by limiting the tricarboxylic acid cycle at the acetyl-CoA node. Finally, the resulting strain exhibited a high NADH level and enabled production of $6.1 \mathrm{~g} / \mathrm{L} n$-butanol with a yield of $0.31 \mathrm{~g} / \mathrm{g}$-glucose and a productivity of $0.21 \mathrm{~g} / \mathrm{L} / \mathrm{h}$.
\end{abstract}

Conclusions: The production efficiency of fermentative products in microbes strongly depends on the intracellular redox state. This work illustrates the flexibility of pyruvate, G6P, and acetyl-CoA nodes at the junction of the central metabolism for engineering. In principle, high production of reduced products of interest can be achieved by individual or coordinated modulation of these metabolite nodes.

Keywords: $n$-Butanol, The redox state, Metabolic engineering, Escherichia coli

\section{Background}

Our daily life is tightly linked to the petroleum-based industries. However, the rising price, the insecure supply, and the environmental concern of fossil fuels have currently overshadowed these industries. Consequently, it provokes the demand for renewable and environmentfriendly fuels and chemicals [1]. The bioprocess production of these chemical commodities appears to be

\footnotetext{
*Correspondence: oleosin91@yahoo.com.tw; ypchao@fcu.edu.tw

${ }^{3}$ Department of Medical Laboratory Science and Biotechnology, China

Medical University, No. 91, Hsueh-Shih Road, Taichung 40402, Taiwan,

Republic of China

${ }^{5}$ Department of Medical Research, China Medical University Hospital,

Taichung 40447, Taiwan, Republic of China

Full list of author information is available at the end of the article
}

appealing [2,3]. Bio-based fuels and chemicals of interest are generally fermentative products of living microbes. The microbial fermentation pathways involve many redox reactions, which usually require $\mathrm{NADH}$ and $\mathrm{NAD}^{+}$ as cofactors. With $\mathrm{NAD}^{+}$as an electron acceptor, the oxidation of sugars produces NADH. NAD ${ }^{+}$is regenerated when intermediate metabolites in the sugar catabolism are subsequently reduced at the expense of NADH. The result of the reductive reactions usually leads to production of ethanol, lactate, and succinate as exemplified in fermentative Escherichia coli [4]. Therefore, maintaining the redox balance of $\mathrm{NADH}$ and $\mathrm{NAD}^{+}$is a key to ensure the continued operation of cellular metabolism under the fermentative condition. 
Fermentative production of $n$-butanol in Clostridium species is a well-known bioprocess [5]. The fermentation process mainly consists of the acidogenesis and the solventogenesis phase [6]. In the acidogenesis stage, the growing Clostridium species ferment glucose to mainly produce acetate and butyrate. Upon reaching the stationary growth phase, the bacteria re-assimilate these organic acids, while acetone, $n$-butanol, and ethanol are produced as end products. In view of the clostridial synthetic pathway, the direct synthesis of $n$-butanol from glucose can cause the NADH/NAD ${ }^{+}$redox imbalance because more NADH is required in the synthetic pathway than that generated in the glycolytic pathway. This may explain why the synthesis of $n$-butanol in Clostridium species proceeds in two stages.

$n$-Butanol is an alternative fuel of potential because its property is superior to ethanol in terms of the energy density, the vapor pressure, and hygroscopicity [7]. Moreover, $n$-butanol can be used for the transportation fuel after blended with gasoline at any concentrations and transported with the existing pipeline infrastructure [8]. These merits make microbial production of $n$-butanol industrially attractive. Many approaches have been proposed for production of $n$-butanol in a variety of surrogate strains [9-12]. However, these attempts are generally discouraged by their low $n$-butanol titer. Recognized as the biotechnology workhorse, $E$. coli has been commonly employed to produce value-added chemicals and biofuels $[13,14]$. Production of $n$-butanol in $E$. coli is proven feasible after introduction of the clostridial synthetic pathway into the strain $[9,15,16]$. In addition, the production titer is improved by manipulating pyruvate dehydrogenase (PDH) and formate dehydrogenase (FDH) in glycolysis to increase the NADH availability in the cell $[15,17,18]$. Recently, we have proposed a new production platform based on two strains: a butyrate-conversion strain and a butyrate-producing strain [19]. The dual-culture system rebuilds a redox-balanced synthetic pathway, which enables effective production of $n$-butanol.

Microbial production of a highly reduced fermentation product such as $n$-butanol remains challenging because there is a limited NADH output from glucose catabolism. This issue was addressed by rerouting the central metabolic pathways in E. coli. The NADH availability is manifested by the interplay of glycolysis, the pentose phosphate (PP) pathway, and the tricarboxylic acid (TCA) cycle involved in central metabolism (Fig. 1). In addition to $\mathrm{PDH}$ and $\mathrm{FDH}$, the enzymes that regulate the metabolite pools at the junction of central metabolism were systematically manipulated to modulate the intracellular NADH. As a result, the engineered strain with the remodeled pathways enabled effective production of n-butanol.

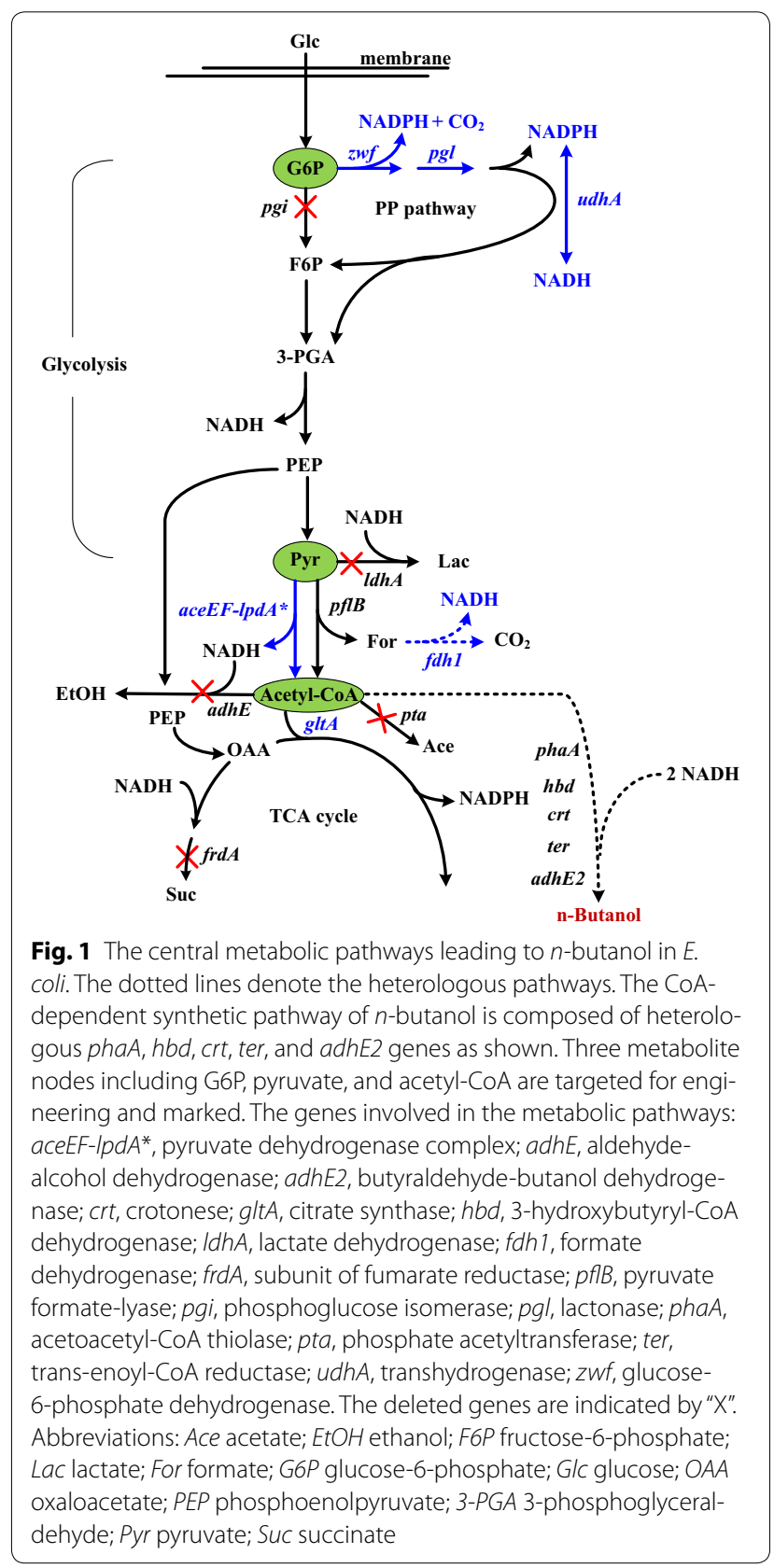

\section{Results and discussion}

Amplification of the pyruvate oxidation pathway

As shown in Fig. 1, the reductive synthesis of one $n$-butanol from one glucose requires more $\mathrm{NADH}$ than that provided in glycolysis. Therefore, the approach of NADH replenishment is expected to favor the fermentative production of $n$-butanol. In this context, the pyruvate node connecting glycolysis and the TCA cycle appears to be a potential target for manipulation. In E. coli, pyruvate is oxidized to acetyl-CoA by a reaction mediated by $\mathrm{PDH}$ under the aerobic growth and by pyruvate formate-lyase 
(PFL) under the fermentative growth [4]. Formate is the reduced product of the PFL reaction. Found in other microbes, FDH such as Candida boidinii fdh and Saccharomyces cerevisiae fdh 1 catalyzes oxidation of formate to $\mathrm{CO}_{2}$ associated with NADH generation [20]. These two genes have been employed in $E$. coli to elevate intracellular NADH, resulting in an increase in $n$-butanol production [17, 21]. Accordingly, S. cerevisiae fdh1 under the control of the trc promoter (Ptrc) without lacO was integrated into strain BuT-8. Strain BuT- 8 was previously constructed with a CoA-dependent pathway of $n$-butanol consisting of the constitutive $\lambda \mathrm{P}_{\mathrm{L}}$ promoter $\left(\mathrm{P} \lambda \mathrm{P}_{\mathrm{L}}\right)$-driven $h b d$, crt, and adhE2 of Clostridium acetobutylicum, phaA of Cupriavidus necator, and ter of Treponema denticola [19]. In addition, the undesired pathways involving endogenous adhE, ldhA, pta, and frdA were removed from this strain to curtail carbon waste and conserve $\mathrm{NADH}$. Equipped with $S$. cerevisiae fdh1, the resulting strain BuT-8-Fdh1 produced $3.1 \mathrm{~g} / \mathrm{L} n$-butanol at $24 \mathrm{~h}$ (Fig. 2a). This production titer accounts for a $25 \%$ increase over that for strain BuT-8 (Table 1).

In contrast to PFL, the PDH reaction generates NADH as the reduced product. Therefore, manipulation of the $\mathrm{PDH}$ level is expected to alter intracellular NADH. This was conducted by fusion of $\mathrm{P} \mathrm{P}_{\mathrm{L}}$ with aceEF operon to enhance the gene expression in strain BuT-8-Fdh1. To render PDH less sensitive to NADH inhibition, the endogenous $\operatorname{lp} d A$ (encoding dihydrolipoamide dehydrogenase) was deleted and a mutation site (E354K) in $l p d A$ was additionally created [22]. The mutant $\operatorname{lp} d A^{*}$ under the control of $\mathrm{P} \lambda \mathrm{P}_{\mathrm{L}}$ was then inserted into strain BuT-8Fdh1 to obtain strain BuT-9. Consequently, strain BuT-9 exhibited 1.3-fold higher PDH activity and $45 \%$ higher NADH level as compared to strain BuT-8 (Table 1). The accumulated pyruvate in strain BuT- 8 was greatly reduced and carbon flux was diverted from the synthetic pathways of byproducts (Table 2). Strain BuT-9 finally produced $4.3 \mathrm{~g} / \mathrm{L} n$-butanol (Fig. $2 \mathrm{~b}$ ), which accounts for a $60 \%$ increase over that in strain BuT-8 (Table 1).

The similar approach has been previously applied for $n$-butanol production in $E$. coli that carries the CoAdependent synthetic pathway. By using C. boidinii fdh, the best strain in their study showed a 1.3-fold increase in $n$-butanol production [17]. In another work, a 1.6fold improvement in the production yield was reported for a strain with enhanced PDH [15]. In addition, it was reported that a strain with the optimal activation of PDH exhibited a $12 \%$ improvement in $n$-butanol production [18]. Further improvement of the strain by optimization of S. cerevisiae fdh1 expression led to a $35.4 \%$ increase in the production titer [18]. These studies were conducted using super-rich TB medium, in contrast to ours which employed M9Y medium. Although displaying a various

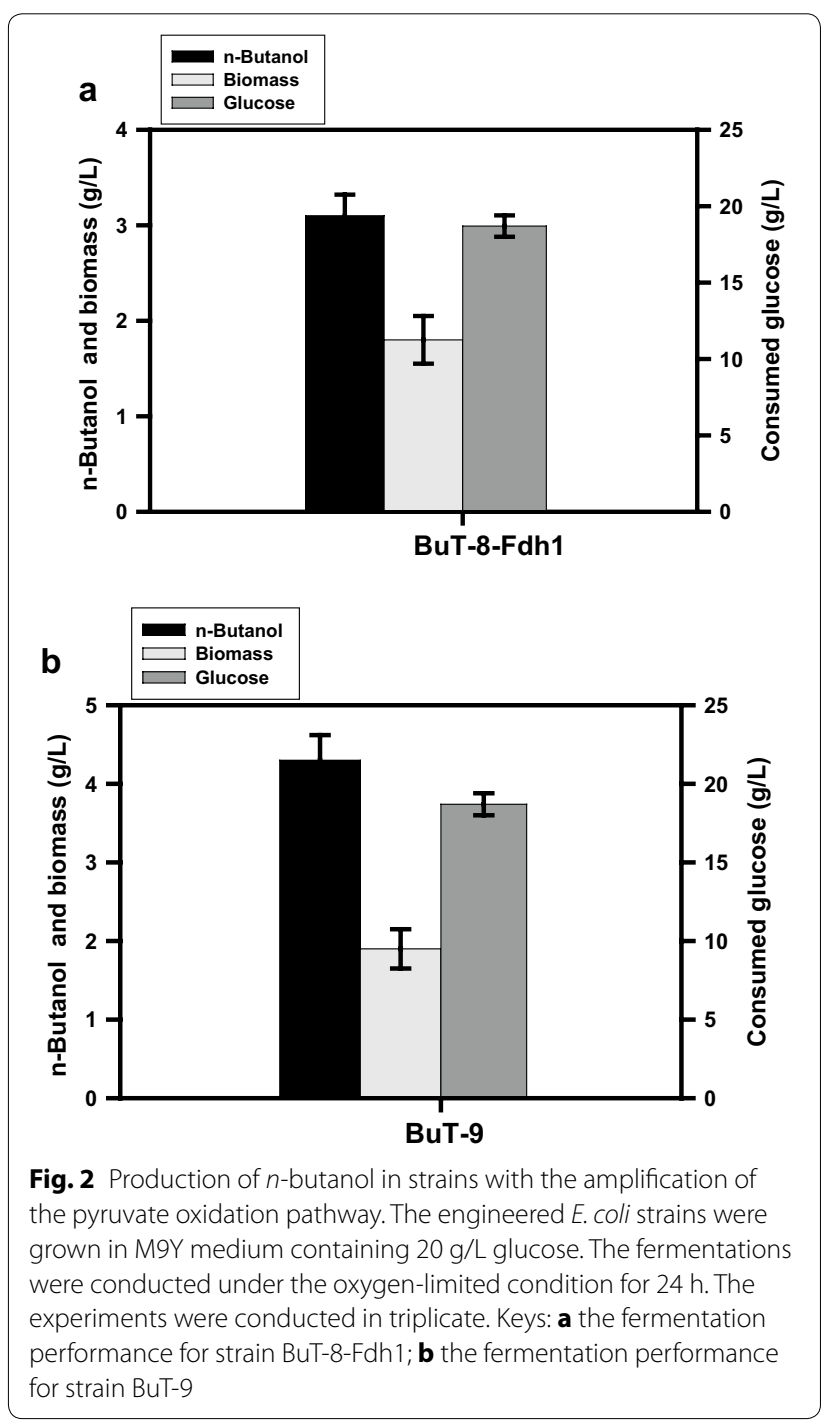

degree of improvement in the $n$-butanol production, aforementioned studies and ours illustrate the feasibility in manipulating the pyruvate node to modulate intracellular NADH.

\section{Amplification of the pentose phosphate pathway}

The glycolytic pathway bifurcates at the glucose-6-phosphate (G6P) node (Fig. 1). With G6P as a starting metabolite, the PP pathway produces precursors for the synthesis of nucleic acids and aromatic amino acids and also provides a major source of NADPH for biosynthesis which is involved in most of the reductive pathways [4]. It is possible to increase NADH availability by manipulation of the G6P node. G6P dehydrogenase (encoded by $z w f$ ) catalyzes the first step in the PP pathway. Therefore, $z w f$ of strain BuT-9 was fused to $\mathrm{P} \mathrm{P}_{\mathrm{L}}$. In E. coli, pyridine nucleotide transhydrogenase (encoded by $u d h A$ ) functions to 
Table 1 Summary of the fermentation kinetics for main engineered strains

\begin{tabular}{|c|c|c|c|c|c|c|c|c|}
\hline \multirow[t]{2}{*}{ Strain } & \multirow[t]{2}{*}{$Y_{B}(g / L)$} & \multirow[t]{2}{*}{$P_{B}(g / L / h)$} & \multirow[t]{2}{*}{$Y_{B / G}(g / g)$} & \multirow[t]{2}{*}{$\mathrm{NADH}(\mu \mathrm{mol} / \mathrm{g}$ cell) } & \multicolumn{4}{|c|}{$\begin{array}{l}\text { Specific enzyme activity } \\
\text { (U/mg protein) }\end{array}$} \\
\hline & & & & & PDH & Zwf & $\mathrm{Pgl}$ & GltA \\
\hline BuT-8 & $2.7^{a}$ & $0.11^{\mathrm{a}}$ & $0.14^{\mathrm{a}}$ & 42.2 & 0.7 & ND & ND & ND \\
\hline BuT-9 & 4.3 & 0.18 & 0.22 & 60.9 & 1.6 & 6.1 & ND & ND \\
\hline BuT-10 & 4.9 & 0.20 & 0.25 & 75.8 & 18.3 & 0.5 & ND & ND \\
\hline BuT-12 & 5.4 & 0.23 & 0.27 & 82.6 & ND & ND & 6.2 & 2.2 \\
\hline BuT-14 & 6.1 & 0.21 & 0.31 & 96.1 & ND & ND & ND & 1.5 \\
\hline
\end{tabular}

a The kinetic data were drawn from the previous report [19]. The development course of producer strains for the $n$-butanol production was shown in Additional file 1: Fig. S1. $U \mu \mathrm{mole} / \mathrm{min}, Y_{B}$ the $n$-butanol titer, ND not determined

Table 2 Carbon recovery of fermentation products for engineered strains during the oxygen-limited growth on glucose

\begin{tabular}{|c|c|c|c|c|c|c|c|c|}
\hline Strain & Pyruvate & Succinate & Ethanol & Lactate & Acetate & Butyrate & Butanol & Total (\%) \\
\hline BuT-8 & 20.7 & 3.5 & 7.8 & 2.7 & 1.5 & 4.1 & 21.9 & 62.2 \\
\hline BuT-9 & 2.6 & 2.1 & 5.2 & 1.8 & 1.2 & 2.7 & 34.8 & 50.4 \\
\hline BuT-10 & 0.01 & 1.1 & 5.2 & 1.5 & 0.8 & 2.0 & 39.6 & 50.2 \\
\hline BuT-12 & Nil & 0.8 & 5.2 & 1.4 & 0.7 & 1.4 & 43.7 & 53.2 \\
\hline BuT-14 & Nil & 0.6 & 2.6 & 1.3 & 0.5 & 1.4 & 49.4 & 55.8 \\
\hline
\end{tabular}

Carbon recovery was calculated as the molar percent of carbon in products per carbon in consumed glucose. Nil, carbon recovery less than 0.01

interconvert NADPH and NADH [23]. Therefore, strain BuT-10 was obtained by further fusion $u d h A$ of strain BuT-9 with $\mathrm{P} \lambda \mathrm{P}_{\mathrm{L}}$ to enhance its expression. As compared to strain BuT-9, strain BuT-10 displayed twofold higher Zwf activity and a $10 \%$ increase in the $n$-butanol production (4.9 g/L) (Table 1; Fig. 3a).

The developed strain is derived from strain BL21 which lacks pgl [24], a gene encoding lactonase that is responsible for the reaction following Zwf in the PP pathway. It is likely that yieK with an annotated function of Pgl is functioning in $E$. coli B strain but less active. Therefore, the carbon flux channeled into the PP pathway by elevated Zwf may be limited at the Pgl-mediated reaction step. To address this issue, the $\mathrm{P} \lambda \mathrm{P}_{\mathrm{L}}$-driven $p g l$ from $E$. coli $\mathrm{K}-12$ strain was re-introduced into strain BuT-10. Finally, the resulting strain (BuT-12) enabled production of $5.4 \mathrm{~g} / \mathrm{L} n$-butanol (Fig. 3b). As compared to strain BuT10, strain BuT-12 displayed a tenfold higher Pgl activity, a $36 \%$ more NADH, and a $25.6 \%$ improvement in the $n$-butanol production (Table 1 ). The approach by enhancing the PP pathway results in more NADH production, which drives more acetyl-CoA into the synthetic pathway of $n$-butanol. This is supported by the observed decrease in pyruvate and succinate (Table 2).

It is apparent that redistribution of carbon flux in glycolysis and the PP pathway can greatly affect intracellular NADH level. Notice that entry of one glucose into the oxidative PP pathway generates two reducing equivalents but wastes one $\mathrm{CO}_{2}$. Nevertheless, strain BuT-12 which is manipulated at the pyruvate and G6P nodes displays a $96 \%$ increase in the NADH level and doubles the $n$-butanol production as compared to strain BuT-8 (Table 1).

\section{Rerouting catabolic pathways of glucose}

According to the central metabolism of E. coli (Fig. 1), glucose catabolism proceeding via the PP pathway generates $85 \%$ more reducing power per gram mole of glucose than that via glycolysis. It seems useful to increase intracellular NADH by diverting the glycolytic flux to the PP pathway. Phosphoglucose isomerase (encoded by pgi) is responsible for isomerization of G6P, and its inactivation makes the PP pathway the primary route of glucose catabolism [25]. Therefore, strain BuT-13 was obtained by deletion of pgi in strain BuT-12. In comparison with strain BuT-12, strain BuT-13 grew poorly (0.31/h vs. $0.5 / \mathrm{h}$ ), while it exhibited a $32 \%$ and $30 \%$ decrease in biomass yield and glucose utilization, respectively (Fig. 4). After fermentation for $30 \mathrm{~h}$, strain BuT-13 was unable to consume all glucose and produced less $n$-butanol $(4.6 \mathrm{~g} / \mathrm{L})$.

Improving NADPH availability was realized by a strain deprived of $p g i$, whereas the strain showed a $47 \%$ decrease in the specific growth rate [26]. The severe growth defect resulting from pgi knockout is attributed to a surplus of NADPH, which in turn perturbs the physiological state of cells [27]. Interestingly, an elevated level of either UdhA or Zwf can lead to growth recovery of 

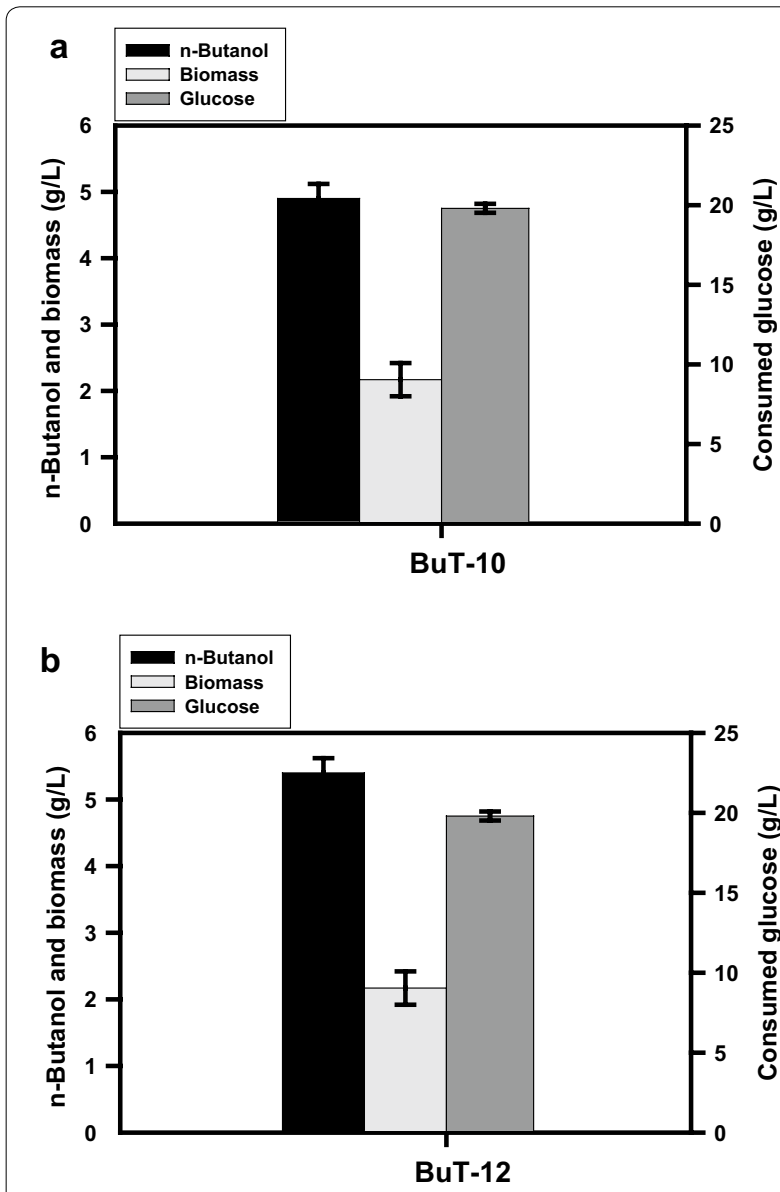

Fig. 3 Production of $n$-butanol in strains with the amplification of the PP pathway. The engineered E. coli strains were grown in M9Y medium containing $20 \mathrm{~g} / \mathrm{L}$ glucose. The fermentations were conducted under the oxygen-limited condition for $24 \mathrm{~h}$. The experiments were conducted in triplicate. Keys: a the fermentation performance for strain BuT-10; b the fermentation performance for strain BuT-12

the pgi-deficient strain by 25 and $68 \%$, respectively [27, 28]. Strain BuT-13 is still afflicted by the growth defect although it exhibits higher UdhA and Zwf activities and harbors the $n$-butanol synthetic pathway that consumes the reducing equivalent. The result implies the presence of an imbalanced redox state in the strain.

In response to the oxygen tension, the TCA cycle operates as either an oxidative pathway or a reductive pathway associated with production of various levels of reducing equivalents [4]. Citrate synthase (encoded by $g l t A$ ) catalyzes the first committed step in the TCA cycle (Fig. 1). The approach to divert carbon flux from the TCA cycle by lowering the GltA activity is expected to conserve acetyl-CoA (the precursor of $n$-butanol) and modulate production of reducing equivalents. It may be helpful to ameliorate the negative impact on the strain

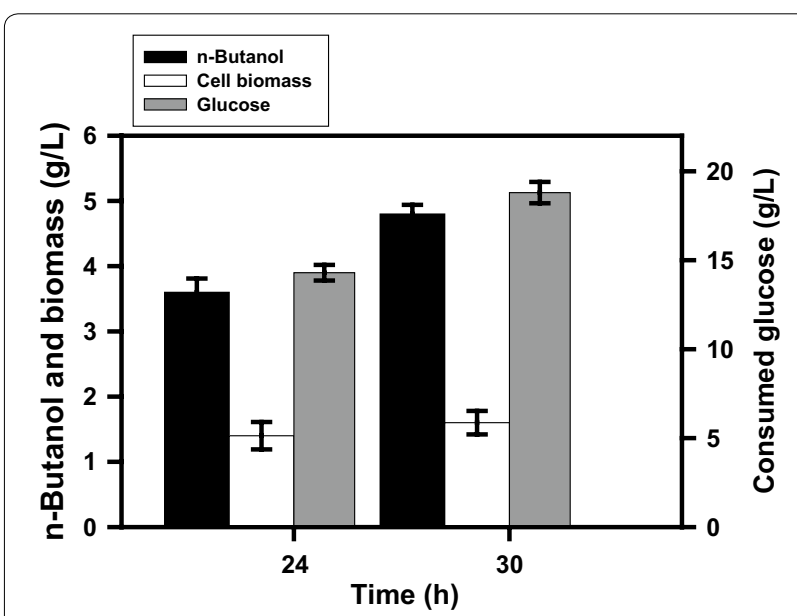

Fig. 4 Production of $n$-butanol in the strain with the glucose catabolism via the PP pathway. Strain BuT-13 grown in M9Y medium containing $20 \mathrm{~g} / \mathrm{L}$ glucose. The fermentations were conducted under the oxygen-limited condition for $30 \mathrm{~h}$. The experiments were conducted in triplicate

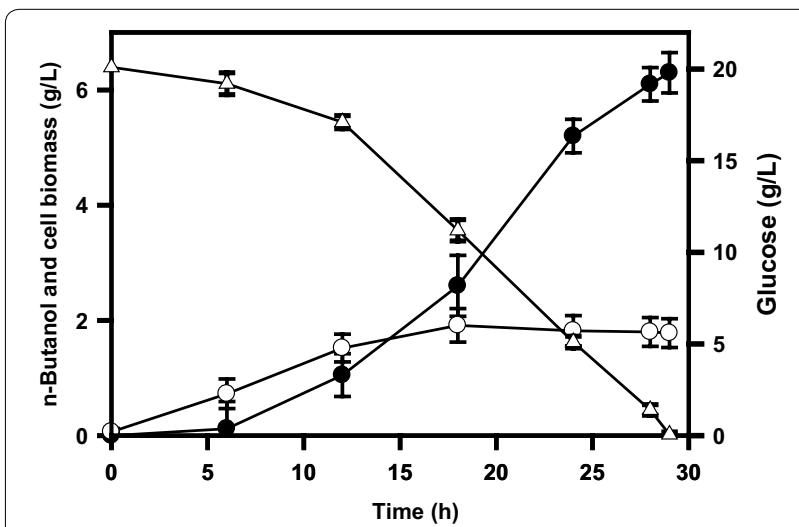

Fig. 5 Time course of $n$-butanol production in the strain with the limited TCA cycle. Strain BuT-14 grown in M9Y medium containing $20 \mathrm{~g} / \mathrm{L}$ glucose. The fermentations were conducted under the oxygen-limited condition. The experiments were conducted in triplicate

imposed by null pgi. This was carried out by replacement of the gltA cognate promoter P2 with lacO site in strain BuT-13. The resulting strain BuT-14 was then cultured and examined for its fermentation performance. Consequently, strain BuT-14 grew almost normally (ca. $0.46 / \mathrm{h}$ ), and its biomass yield was comparable to strain BuT-12. All fermentation byproducts were significantly reduced in strain BuT-14 which consequently produced $6.1 \mathrm{~g} / \mathrm{L} n$-butanol at $29 \mathrm{~h}$ (Table 2; Fig. 5). As expected, strain BuT-14 exhibited $32 \%$ less GltA activity and $16 \%$ more NADH as compared to strain BuT-12. Direction of carbon flux through the PP pathway by pgi deletion 
increases the reducing equivalent, whereas prevention of carbon flux from entering the TCA cycle by lowering GltA reduces the reducing equivalent. It leads to a net outcome of a moderate increase in NADH, which suffices the need for the synthesis of $n$-butanol. As a result, a redox-balanced state is established in strain BuT-14 that recovers from the null $p g i$-induced growth defect. It was intriguing to learn the response of this strain to the act of further lowering the GltA activity. Strain BuT-14-A was thus obtained by integration of $l a c I^{\mathrm{Q}}$ into strain BuT-14. Consequently, strain BuT-14-A displayed 50 \% less GltA activity than strain BuT-12 (Table 1). The strain exhibited a poor growth and consumed only $40 \%$ glucose associated with the $n$-butanol production of $1.8 \mathrm{~g} / \mathrm{L}$ at $30 \mathrm{~h}$ (data not shown). It was reported that the growth of $E$. coli on glucose remains unaffected by a $90 \%$ decrease in the GltA activity [29]. In contrast, the growth of the $n$-butanol-producing strain that lacks $p g i$ on glucose is closely linked to the GltA activity. By modulation of the GltA activity, the producer strain enables recovery from the growth defect caused by null pgi. It is likely that the alteration of the GltA activity perturbs the intracellular redox state of the strain. Apparently, the engineered strain displays high susceptibility to the intracellular redox state and optimal adjustment of GltA activity is necessary to ensure the superior performance of the strain.

Recruitment of FDH and enhanced expression of PDH have been proposed to increase NADH availability favoring the $n$-butanol production in E. coli. In one study, the approach by recruiting FDH achieved the $n$-butanol productivity $\left(\mathrm{P}_{\mathrm{B}}\right)$ of $0.2 \mathrm{~g} / \mathrm{L} / \mathrm{h}$ and the conversion yield on glucose $\left(\mathrm{Y}_{\mathrm{B} / \mathrm{G}}\right)$ of $0.36 \mathrm{~g} / \mathrm{g}$ [17]. An alternative study by manipulating PDH reported $\mathrm{P}_{\mathrm{B}}$ of $0.065 \mathrm{~g} / \mathrm{L} / \mathrm{h}$ and $\mathrm{Y}_{\mathrm{B} / \mathrm{G}}$ of $0.19 \mathrm{~g} / \mathrm{g}$ [15]. In addition, $\mathrm{P}_{B}$ of $0.26 \mathrm{~g} / \mathrm{L} / \mathrm{h}$ and $\mathrm{Y}_{\mathrm{B} / \mathrm{G}}$ of $0.27 \mathrm{~g} / \mathrm{g}$ were obtained by optimization of both FDH and PDH activity in a recent work [18]. All these studies were conducted with super-rich TB medium, and plasmids were employed for the episomal expression of multiple target genes to increase their expression levels, which is important to obtain the observed effect. Therefore, the discrepancy in the fermentation production by these reports is likely attributed to various expression levels of the cloned genes. However, plasmid maintenance is known to impose a metabolic burden on cells, thus resulting in a reduced growth rate and perturbation of the cell physiology [30]. A metabolic load is additionally imposed on E. coli after the forced expression of the plasmid-borne genes, which suppresses the primary carbon and energy metabolism of cells [31]. The use of TB medium $(12 \mathrm{~g} / \mathrm{L}$ tryptone, $24 \mathrm{~g} / \mathrm{L}$ yeast extract, $2.31 \mathrm{~g} / \mathrm{L}$ $\mathrm{KH}_{2} \mathrm{PO}_{4}, 12.54 \mathrm{~g} / \mathrm{L} \mathrm{KHPO}_{4}, 4 \mathrm{~mL} / \mathrm{L}$ glycerol) is expected to improve the cell growth under the anaerobic condition and helps to ameliorate the plasmid-induced stress on cells. It should be noted that components other than glucose in the TB medium could contribute $15 \%$ of the $n$-butanol production according to the previous study [17]. Therefore, these reported yields based on glucose may be re-estimated. In contrast, the engineered strains were free of plasmids and grown on the M9Y medium with glucose in this study. First, the starter strain BuT-8 displayed $\mathrm{P}_{\mathrm{B}}$ of $0.11 \mathrm{~g} / \mathrm{L} / \mathrm{h}$ and $\mathrm{Y}_{\mathrm{B} / \mathrm{G}}$ of $0.14 \mathrm{~g} / \mathrm{g}$ (Table 1 ). With both enhanced FDH and $\mathrm{PDH}$, strain BuT-9 achieved $\mathrm{P}_{\mathrm{B}}$ of $0.18 \mathrm{~g} / \mathrm{L} / \mathrm{h}$ and $\mathrm{Y}_{\mathrm{B} / \mathrm{G}}$ of $0.22 \mathrm{~g} / \mathrm{g}$. Finally, strain BuT-14 was developed by rerouting of the glycolytic flux through the PP pathway and further reducing GltA activity in the TCA cycle. It exhibited 2.3-fold higher NADH and $n$-butanol production titer than the starter strain BuT-8. Notice that $n$-butanol was not produced for strain BuT-14 grown on M9Y medium without glucose (data not shown). Therefore, the $n$-butanol production in the strain comes merely from glucose. As a result, strain BuT-14 displayed $\mathrm{P}_{\mathrm{B}}$ of $0.21 \mathrm{~g} / \mathrm{L} / \mathrm{h}$ and $\mathrm{Y}_{\mathrm{B} / \mathrm{G}}$ of $0.31 \mathrm{~g} / \mathrm{g}$. In theory, $0.85 \mathrm{~mol}$ instead of $1 \mathrm{~mol}$ $n$-butanol per mole glucose is produced in the glucose catabolism via the PP pathway due to $\mathrm{CO}_{2}$ loss, which leads to the theoretical yield of $n$-butanol as $0.35 \mathrm{~g} / \mathrm{g}$. Accordingly, $n$-butanol remains the main product for strain BuT-14 (Table 2) with $\mathrm{Y}_{\mathrm{B} / \mathrm{G}}$ reaching $89 \%$ of the theoretical. It is feasible to recycle $\mathrm{CO}_{2}$ in E. coli with the expression of Synechococcus ribulose-1,5-bisphosphate carboxylase/oxygenase (Rubisco) and phosphoribulokinase [32]. The issue of increasing $\mathrm{Y}_{\mathrm{B} / \mathrm{G}}$ may be addressed by recruitment of the Rubisco-based $\mathrm{CO}_{2}$ recycling system in strain BuT-14. Overall, it indicates that our proposed approach is effective for production of the highly reduced product without the need for a super-rich medium.

\section{Conclusions}

The intracellular redox state in microbes is recognized as a key factor determining the production efficiency of fermentation products. The current work illustrates that pyruvate, G6P, and acetyl-CoA at the junction of the central catabolism are flexible for engineering. It is known that the enzymes, such as PDH and GltA, involved in the use of these metabolite nodes are subjected to the physiological control mediated by the effector metabolites [4]. Nevertheless, genetic manipulation of these metabolite nodes can lead to the redistribution of carbon flux, which in turn alters the cellular redox state. In principle, these metabolite nodes can be individually or coordinately modulated to fulfill the intracellular need for high production of reduced products of interest. 


\section{Methods}

\section{Bacterial culturing}

The method for bacterial culturing under the oxygenlimited condition essentially followed the previous report [19]. The seeding cultures were prepared by growing $E$. coli strains on Luria-Bertani medium [33] with $2 \mathrm{~g} / \mathrm{L}$ glucose overnight. The cell density was measured turbidimetrically at $550 \mathrm{~nm}\left(\mathrm{OD}_{550}\right)$. The overnight culture was inoculated into capped Erlenmeyer flasks $(125 \mathrm{~mL})$ containing $50 \mathrm{~mL}$ M9Y medium $\left(6 \mathrm{~g} / \mathrm{L} \mathrm{Na}_{2} \mathrm{HPO}_{4}, 3 \mathrm{~g} / \mathrm{L}\right.$ $\mathrm{KH}_{2} \mathrm{PO}_{4}, 0.5 \mathrm{~g} / \mathrm{L} \mathrm{NaCl}, 1 \mathrm{~g} / \mathrm{L} \mathrm{NH} \mathrm{NH}_{4} \mathrm{Cl} 1 \mathrm{mM} \mathrm{MgSO}$, $0.1 \mathrm{mM} \mathrm{CaCl}, 10 \mathrm{mg} / \mathrm{L}$ vitamin $\mathrm{B} 1,5 \mathrm{~g} / \mathrm{L}$ yeast extract) with $20 \mathrm{~g} / \mathrm{L}$ glucose to attain the initial cell density at $\mathrm{OD}_{550}$ of 0.2 . The shake flask cultures were maintained in an orbital shaker set at $100 \mathrm{rpm}$.

\section{Strain construction}

The strains and primers applied in this study are listed in Table 3. Genomic insertion of $f d h 1$ into strain BuT- 8 was constructed previously [34]. In brief, Ptrc-driven fdh1 of S. cerevisiae was amplified from plasmid pTrc-Fdh1 [21] by PCR with primers RC12171/RC12314. After digestion with BamHI, the PCR DNA was spliced into BamHINruI of plasmid pP21-Km to obtain plasmid pP21-Fdh1. Based on our reported methods, the DNA containing Ptrc- $f d h 1$ was then integrated into $E$. coli using plasmid pP21-Fdh1, and the inserted kanamycin-resistant marker in the strain was later removed [35]. Strain BuT-8 with $f d h 1$ was renamed BuT-8-Fdh1.

Plasmid pMCS-lpdA was obtained by PCR-amplification of $\operatorname{lpdA}$ from strain BL21 with primers RC12154/ RC12155 and subsequent incorporation into NdeI-XhoI of plasmid pMCS-5. The E354K mutation was introduced into $\operatorname{lpdA}$ on plasmid pMSC-lpdA by the site-directed mutagenesis using primers RC12215/RC12216. The mutant $l p d A$ ( $l p d A *)$ was confirmed by DNA sequencing and removed from plasmid pMCS-lpdA by NdeI$X$ hoI. The recovered $l p d A *$ was subcloned into plasmid pLoxKm-PR [36] which carries a cassette of $\mathrm{P} \lambda \mathrm{P}_{\mathrm{L}}$ fused with $\mathrm{LE}^{*}-k a n-\mathrm{RE}^{*}\left(\mathrm{LE}^{*}-k a n-\mathrm{RE}^{*}-\mathrm{P} \lambda \mathrm{P}_{\mathrm{L}}\right)$. The resulting plasmid pLoxKm-lpdA* contains the $\mathrm{LE}^{*}-k a n-\mathrm{RE}^{*}-\mathrm{P} \lambda \mathrm{P}_{\mathrm{L}^{-}}$ regulated $l p d A^{*}\left(\mathrm{LE}^{*}-k a n-\mathrm{RE} * \mathrm{P} \lambda \mathrm{P}_{\mathrm{L}}-l p d A^{*}\right)$. Meanwhile, the upstream region of $l p d A$ was amplified by PCR with primers RC12289/RC12290 and spliced into BamHI-SacI of plasmid pBluescript to produce plasmid pBlue-ac. The DNA containing $L E^{*}-k a n-\mathrm{RE}^{*}-\mathrm{P} \lambda \mathrm{P}_{\mathrm{L}}-l p d A *$ was recovered from plasmid pLoxKm-lpdA* by BamHI-XhoI and then incorporated into plasmid pBlue-ac to give plasmid pBlue-ac/lpdA*. In addition, PCR was conducted on plasmid pBlue-ac/lpdA* with primers RC11210/ RC12331. The PCR DNA was digested with EcoRI and self-ligated to give plasmid pBlue-Ac-lpd, which carries $l p d A$ interrupted with LE*-kan-RE*. To knockout $l p d A$,
Table 3 The strains and primers applied in this study

\begin{tabular}{|c|c|c|}
\hline & Characteristics & Source \\
\hline \multicolumn{3}{|l|}{ Strains } \\
\hline \multirow[t]{3}{*}{ BuT-8 } & $\triangle$ frdA $\phi 80 a t t B:: P \lambda P_{L}-c r t$ & \\
\hline & $\triangle a d h E:: \phi 80 a t t B:: P \lambda P_{L}-p h a-h b d$ & \\
\hline & $\triangle l d h A:: \lambda a t t B:: P \lambda P_{L}-a d h E 2$ & 19 \\
\hline BuT-8-Fdh1 & as P21attB:: Ptac-fdh1 & 34 \\
\hline BuT-9 & $\begin{array}{l}\text { as But-8-Fdh } \triangle l p d A \lambda a t t B:: P \lambda P_{L}-I p d A^{*} \\
\quad P \lambda P_{L}-a c e E F\end{array}$ & This study \\
\hline BuT-10 & as But-9 $P \lambda P_{L}-z w f P \lambda P_{L}-U d h A$ & This study \\
\hline BuT-12 & as But-10 $\triangle a t o D:: P \lambda P_{L}-p g l$ & This study \\
\hline BuT-13 & as But-12 $\Delta p g i$ & This study \\
\hline BuT-14 & as But-13 lacO-gltA & This study \\
\hline \multicolumn{3}{|l|}{ Primers } \\
\hline RC10178 & ATAAGGATCCATATCTAACACCGTGCGTG & \\
\hline RC11210 & CACACCATATGTTAGAATTCATTACCTTCG & \\
\hline RC11403 & TTTGCGGTACCAAGCCCTTTGCAAATTGC & \\
\hline RC11404 & CAGCAGAGCTCGAATGGATCGCGTTATC & \\
\hline RC11405 & AGAATCATATGGCGGTAACGCAAACAG & \\
\hline RC11406 & CTTAAGGATCCTAACCCGGTACTTAAGCCAG & \\
\hline RC11407 & CGTAAGGTACCTGACGCATGCGCGTTTG & \\
\hline RC11408 & ACTTAGAGCTCTAAATGCGGCTTCCACCAG & \\
\hline RC11409 & GCCCTCATATGCCACATTCCTACGATTAC & \\
\hline RC11410 & TGTTCGGATCCATAAAAGCAACAGAATGGTAAC & \\
\hline RC11417 & CCAAGCCCTTTGCAAATTGC & \\
\hline RC11418 & CTCGAATGGATCGCGTTATC & \\
\hline RC11419 & CCTGACGCATGCGCGTTTG & \\
\hline RC11420 & CTAAATGCGGCTTCCACCAG & \\
\hline RC12058 & AATAACATATGTCAGAACGTTTCCCAAATG & \\
\hline RC12059 & CTATCTCTAGACGTTGAGTTTTCTGGAACC & \\
\hline RC12060 & CCAGTTCGAGGTCTTTTTTCG & \\
\hline RC12085 & TATGGGGTACCAGTTCGAGGTCTTTTTTTCG & \\
\hline RC12086 & CAATGGAGCTCTGCTTCATCTGCTAAGG & \\
\hline RC12154 & GCGATATCGTCGGTCAACC & \\
\hline RC12155 & TGAGAAGCTTCAGTCCGCATCACCAGAG & \\
\hline RC12171 & GCAAGCTTATTTCTTCTGTCCATAAGC & \\
\hline RC12215 & GTCCATCGCCTATACCAAACCAGAAGTTGCATG & \\
\hline RC12216 & CATGCAACTTCTGGTTTGGTATAGGCGATGGAC & \\
\hline RC12288 & AACTGCTCGAGTTACTTCTTCTTCGCTTTCG & \\
\hline RC12289 & AAGTGGATCCATACCCGTCGTCTTTCAGG & \\
\hline RC12290 & CCATGAGCTCGGCTTTTTTTCTGGTAATCTC & \\
\hline RC12314 & TCTGGGGATCCTTCTGAAATGAGCTGTTGAC & \\
\hline RC12331 & ACTCTCGAATTCTGGTCGTCCTATCGCTTC & \\
\hline RC13001 & TTGAATTCCGCCTTTAAAGATCGCCATG & \\
\hline RC13034 & CATCTCACCAGATATCATGC & \\
\hline RC13035 & AATCGGAGCTCGAAAGTGAACTGTTTGG & \\
\hline RC13195 & ATCTTCCCGGGCGGAATTCATTACCGTTC & \\
\hline RC13196 & $\begin{array}{l}\text { GAAATTGTTATCCGCTCACAATTCCGGGTACC- } \\
\text { CAATTC }\end{array}$ & \\
\hline RC13197 & CAGCAAAATACCTTCATCACC & \\
\hline RC13198 & TTCAGGGGAAGAGAGGCTG & \\
\hline RC13199 & TCAATGGGCCCACACTGTTACATAAGTTAATC & \\
\hline
\end{tabular}


Table 3 continued

\begin{tabular}{lll}
\hline & Characteristics & Source \\
\hline RC13200 & TTAATGTCGACGATTGCTAAGTACTTGATTCG & \\
RC13201 & GGTACCCAGAAGCCACAG \\
RC13292 & ATCCCGGGAAGCAAACAGTTTATATCGC & \\
RC13293 & ATCTCGAGTAGTGTGCGTAACCACCAC & \\
RC14025 & GAGGAATCTGTAGGCTGGAGCTGCTTC & \\
RC14026 & AACGGTCGACATGGGAATAGCCATGG & \\
\hline
\end{tabular}

the truncated $\operatorname{lpd} A$ was amplified from plasmid pBlueAc-lpd by PCR with primers RC12288/RC12290 and electroporated into $E$. coli following our protocol. Finally, the DNA containing $\mathrm{P} \lambda \mathrm{P}_{\mathrm{L}}-l p d A^{*}$ was amplified from plasmid pBlue-ac/lpdA* by PCR with primers RC10178/ RC12288 and then restricted by BamHI. Plasmid pLamLpdA* was obtained by incorporation of the PCR DNA into BamHI-EcoRV of plasmid pLam-Crt [36]. Similarly, the DNA containing $\mathrm{P} \lambda \mathrm{P}_{\mathrm{L}}-l p d A^{*}$ was integrated into $E$. coli followed by removal of the inserted marker [21].

To enhance the expression of endogenous genes, $\mathrm{P} \lambda \mathrm{P}_{\mathrm{L}}$ was placed in front of the structural genes with their cognate promoters intact. This was carried out as follows: First, the upstream region and $5^{\prime}$-end structural regions of $z w f, u d h$, and aceE were amplified from strain BL21 by PCR with primers RC11403/RC11404, RC11407/ RC11408, and RC12085/RC12086, respectively. Each PCR DNA was digested by KpnI-SacI and incorporated into plasmid pBluescript to obtain plasmid pBlue-zwf, pBlue-udhA, and pBlue-aceE. Secondly, the NdeI-BamHI site was introduced into plasmid pBlue-zwf and pBlueudhA by PCR with primers RC11405/RC11406 and RC11409/RC11410 while the NdeI-XbaI site into plasmid pBlue-aceE with primers RC12058/RC12059. The $\mathrm{LE}^{*}$-kan-RE*-P $\lambda \mathrm{P}_{\mathrm{L}}$ cassette was recovered from plasmid pLoxKm-PR by NdeI-BamHI or NdeI-XbaI digestion and then incorporated into plasmid pBlue-zwf, pBlue-udhA, and pBlue-aceE to obtain plasmid pPR-zwf, pPR-udhA, and pPR-aceE. Finally, the PCR DNAs were amplified from plasmid pPR-zwf, pPR-udhA, and pPR-aceE with primers RC11417/RC11418, RC11419/RC11420, and RC12060/RC12086, respectively. These passenger DNAs were individually integrated into the strain by electroporation according to the reported method [21]. The associated marker was finally rescued.

To obtain $p g l$, the gene was amplified from strain MG1655 with primers RC13292/RC13293. After cleavage by EcoRV-SacI, the PCR DNA and plasmid pBluescript were spliced together to give plasmid pBlue-pgl. The pgl-containing DNA was recovered by SmaI-XhoI and incorporated into plasmid pLoxKm-PL. The construction resulted in plasmid pSPL-pgl, which fuses
$\mathrm{LE} *-k a n-\mathrm{RE}^{*}-\mathrm{P} \lambda \mathrm{P}_{\mathrm{L}}$ with pgl. The $\mathrm{LE} *-k a n-\mathrm{RE}^{*}-\mathrm{P} \lambda \mathrm{P}_{\mathrm{L}}$ pgl DNA was amplified by PCR with primers RC13001/ RC13293. Plasmid pAto-pgl was obtained by incorporation of the PCR DNA into EcoRI-NruI of plasmid pSPL-atoD [36]. Similarly, the passenger DNA was amplified from plasmid pSPL-atoD with primers RC13034/ RC13035 and then electroporated to the strain. Later removal of the inserted marker was carried out.

To modulate the gltA expression, its P2 promoter was replaced with lacO. This was done in several steps. First, lacO was created in plasmid pLoxKm-PR by PCR with primers RC13195/RC13196. After cleavage by SmaI, the PCR DNA was self-ligated to produce plasmid pLoxCm$\mathrm{LacO}$, which carries the fusion of $\mathrm{LE}^{*}-k a n-\mathrm{RE}^{*}-l a c O$. Secondly, the DNA containing the upstream region and 5 -end structural sequence of gltA was amplified from strain BL21 by PCR with primers RC13197/RC13198. Plasmid pBlue-GltA was generated by incorporation of the PCR DNA into KpnI-SmaI of plasmid pBluescript. Moreover, the ApaI-SalI site was introduced into plasmid pBlue-GltA by PCR with primers RC13199/RC13200. The $\mathrm{LE}^{*}$-kan-RE*-lacO cassette was recovered from plasmid pLoxCm-LacO by ApaI-SalI and incorporated into plasmid pBlue-GltA to give plasmid pBlue-GltO. Finally, the FRT-Cm-FRT cassette was amplified from plasmid pKD3 by PCR with primers RC14025/RC14026. The LE*kan-RE* cassette was replaced by FRT-Cm-FRT by incorporation of the PCR DNA into EcoRI-SalI of plasmid pBlue-gltO, leading to plasmid pB-gltO-Cm. The passenger DNA was amplified from plasmid pB-gltO-Cm with primers RC13197/RC13201 and then electroporated to the strain in a similar way.

\section{Analytical method}

The analytical method essentially followed our reported protocol [19]. Glucose was measured by high-performance liquid chromatography (HPLC) with Reflective Index RID-10A (Shimadzu, Japan). $n$-Butanol was determined by Gas Chromatograph Trace 1300 (Thermo Scientific, USA).

The intracellular NADH level was measured by using the fluorescent NAD/NADH detection kit (Cell Technology, USA). The assay procedure exactly followed the manufacturer's instruction. In brief, bacterial cultures were harvested by centrifugation and the cell pellets were resuspended in $200 \mu \mathrm{L}$ NADH extraction buffer plus $200 \mu \mathrm{L}$ lysis buffer. The mixture was kept at $60{ }^{\circ} \mathrm{C}$ for $20 \mathrm{~min}$. After centrifugation, the supernatant was recovered and mixed with the reaction reagent for the measurement. The reaction was kept in dark at room temperature for $1 \mathrm{~h}$. The NADH level was then measured with the excitation at $530-570 \mathrm{~nm}$ and emission at 590-600 nm. 


\section{Enzyme activity assay}

Bacterial cultures were harvested by centrifugation and the cell pellets were resuspended in $1 \mathrm{~mL}$ solution buffer. Cells were disrupted by sonication after centrifugation. The supernatant was saved as the cell-free extract (CFX). The total protein content in CFX was determined using Bio-Rad protein assay kit. The activity of pyruvate dehydrogenase was determined by monitoring the reduction of $\mathrm{NAD}^{+}$at $340 \mathrm{~nm}$ at room temperature according to the previous report [15]. The reaction solution $(1 \mathrm{~mL})$ contains $50 \mathrm{mM}$ potassium phosphate ( $\mathrm{pH} 7.9), 5 \mathrm{mM}$ sodium pyruvate, $1.3 \mathrm{mM}$ CoA, $2 \mathrm{mM} \mathrm{NAD}^{+}, 0.5 \mathrm{mM}$ thiamine pyrophosphate, and $5 \mathrm{mM} \mathrm{MgCl}_{2}$. To start the reaction, $100 \mu \mathrm{L}$ CFX was added to the solution. The activity of glucose-6-phosphate dehydrogenase was determined by monitoring the reduction of $\mathrm{NADP}^{+}$at $340 \mathrm{~nm}$ following the reported protocol [37]. The reaction solution $(1 \mathrm{~mL})$ is composed of $2 \mathrm{mM}$ glucose-6-phosphate, $0.67 \mathrm{mM} \mathrm{NADP}{ }^{+}, 10 \mathrm{mM} \mathrm{MgCl}_{2}$, and $50 \mathrm{mM}$ Tris $-\mathrm{HCl}(\mathrm{pH} 7.5)$. The reaction was initiated by adding $100 \mu \mathrm{L} \mathrm{CFX}$ to the solution at $30{ }^{\circ} \mathrm{C}$. The method for measuring the activity of lactonase was similar to the glucose-6-phosphate dehydrogenase activity assay [38]. The reaction solution comprises $50 \mu \mathrm{M}$ glucose-6-phosphate, $0.5 \mathrm{mM} \mathrm{NADP}^{+}, 50 \mathrm{mM}$ Tris- $\mathrm{HCl}, 10 \mathrm{mM} \mathrm{MgCl}_{2}$, and $50 \mathrm{mM}$ Tris- $\mathrm{HCl}$ (pH 7.5). In addition, the citrate synthase activity was measured as reported previously [29]. The composition of the assay solution includes $0.1 \mathrm{mM}$ acetyl-CoA, $0.5 \mathrm{mM}$ oxaloacetate, $0.2 \mathrm{mM} 5^{\prime} 5$-dithiobis(2-nitrobenzoic acid), and $50 \mathrm{mM}$ Tris- $\mathrm{HCl}$ (pH 7.5).

\section{Additional file}

Additional file 1: Figure S1. The development course of E. coli strains for the fermentative production of n-butanol.

\begin{abstract}
Abbreviations
PP: pentose phosphate; G6P: glucose-6-phosphate; PDH: pyruvate dehydrogenase; FDH: formate dehydrogenase; TCA: tricarboxylic acid; PFL: pyruvate formate-lyase; HPLC: high-performance liquid chromatography; CFX: cell-free extract; Ace: acetate; EtOH: ethanol; F6P: fructose-6-phosphate; Lac: lactate; For: formate; Glc: glucose; OAA: oxaloacetate; PEP: phosphoenolpyruvate; 3PGA: 3-phosphoglyceraldehyde; Pyr: pyruvate; Suc: succinate.
\end{abstract}

\begin{abstract}
Authors' contributions
MS constructed bacterial strains and performed fermentation; SYL analyzed data and drafted the manuscript; ZWW helped gene cloning; CJC and YPC assisted in the design of the study and revised the manuscript. All authors read and approved the final manuscript.
\end{abstract}

\footnotetext{
Author details

${ }^{1}$ Department of Chemical Engineering, Feng Chia University, 100 Wenhwa Road, Taichung 40724, Taiwan, Republic of China. ${ }^{2}$ Department of Chemical Engineering, National Chung Hsing University, Taichung 402, Taiwan, Republic of China. ${ }^{3}$ Department of Medical Laboratory Science and Biotechnology, China Medical University, No. 91, Hsueh-Shih Road, Taichung 40402, Taiwan, Republic of China. ${ }^{4}$ Department of Health and Nutrition Biotechnology, Asia University, Taichung 41354, Taiwan, Republic of China. ${ }^{5}$ Department
}

of Medical Research, China Medical University Hospital, Taichung 40447, Taiwan, Republic of China.

\section{Acknowledgements}

This work is supported by Taiwan Ministry of Science and Technology (MOST 102-2221-E-039-014-MY3, 104-2221-E-035-072, 104-2623-E-035-004-ET, and 104-2622-E-035-011-C(1).

\section{Competing interests}

The authors declare that they have no competing interests.

Received: 17 November 2015 Accepted: 19 February 2016

Published online: 18 March 2016

\section{References}

1. Schubert C. Can biofuels finally take center stage? Nat Biotechnol. 2006;24:777-84.

2. Hermann BG, Blok K, Patel MK. Producing bio-based bulk chemicals using industrial biotechnology saves energy and combats climate change. Environ Sci Technol. 2007;41:7915-21.

3. Stephanopoulos $\mathrm{G}$. Challenges in engineering microbes for biofuels production. Science. 2007;315:801-4.

4. White D. The physiology and biochemistry of prokaryotes. 3rd ed. New York: Oxford University Press; 2007.

5. Jones DT, Woods DR. Acetone-butanol fermentation revisited. Microbiol Rev. 1986:50:484-524.

6. Lee SY, Park JH, Jang SH, Nielsen LK, Kim J, Jung KS. Fermentative butanol production by Clostridia. Biotechnol Bioeng. 2008;101:209-28.

7. Mussatto S, Dragone G, Guimarães PM, Silva JP, Carneiro LM, Roberto IC, et al. Technological trends, global market, and challenges of bio-ethanol production. Biotechnol Adv. 2010;28:817-30.

8. Dürre P. Biobutanol: an attractive biofuel. Biotechnol J. 2007;2:1525-34.

9. Atsumi S, Cann AF, Connor MR, Shen CR, Smith KM, Brynildsen MP, et al. Metabolic engineering of Escherichia coli for 1-butanol production. Metab Eng. 2008;10:305-11.

10. Berezina OV, Zakharova NV, Brandt A, Yarotsky SV, Schwarz WH, Zverlov V. Reconstructing the clostridial $n$-butanol metabolic pathway in Lactobacillus brevis. Appl Microbiol Biotechnol. 2010;87:635-46.

11. Nielsen DR, Leonard E, Yoon SH, Tseng HC, Yuan C, Prather KL. Engineering alternative butanol production platforms in heterologous bacteria. Metab Eng. 2009;11:262-73.

12. Steen EJ, Chan R, Prasad N, Mayers S, Petzold CJ, Redding A, et al. Metabolic engineering of Saccharomyces cerevisiae for the production of n-butanol. Microb Cell Fact. 2008;7:36.

13. Clomburg JM, Gonzalez R. Biofuel production in Escherichia coli: the role of metabolic engineering and synthetic biology. Appl Biochem Biotechnol. 2010;86:419-34.

14. Yu C, Cao Y, Zou H, Xian M. Metabolic engineering of Escherichia coli for biotechnological production of high-value organic acids and alcohols. Appl Biochem Biotechnol. 2011;89:573-83.

15. Bond-Watts BB, Bellerose RJ, Chang MCY. Enzyme mechanism as a kinetic control element for designing synthetic biofuel pathways. Nat Chem Biol. 2011;7:222-7

16. Lim HG, Lim JH, Jung GY. Modular design of metabolic network for robust production of $n$-butanol from galactose-glucose mixtures. Biotechnol Biofuels. 2015;8:137

17. Shen CR, Lan El, Dekishima Y, Baez A, Cho KM, Liao JC. Driving forces enable high-titer anaerobic 1-butanol synthesis in Escherichia coli. Appl Environ Microbial. 2011;77:2905-15.

18. Lim JH, Seo SW, Kim SY, Jung GY. Model-driven rebalancing of the intracellular redox state for optimization of a heterologous $n$-butanol pathway in Escherichia coli. Metab Eng. 2013;20:56-62.

19. Saini M, Chen MH, Chiang CJ, Chao YP. Potential production platform of n-butanol in Escherichia coli. Metab Eng. 2015;27:76-82.

20. Berríos-Rivera SJ, Bennett GN, San KY. The effect of increasing NADH availability on the redistribution of metabolic fluxes in Escherichia coli chemostat cultures. Metab Eng. 2002;4:230-7. 
21. Chiang CJ, Saini M, Lee HM, Wang ZW, Chen PT, Chao YP. Genomic engineering of Escherichia coli by the phage attachment site-based integration system with mutant loxP sites. Proc Biochem. 2012;47:2246-54.

22. Kim Y, Ingram LO, Shanmugam KT. Dihydrolipoamide dehydrogenase mutation alters the NADH sensitivity of pyruvate dehydrogenase complex of Escherichia coli K-12. J Bacteriol. 2008;190:3851-8.

23. Canonaco F, Hess AT, Heri S, Wang T, Szyperski T, Sauer U. Metabolic flux response to phosphoglucose isomerase knock-out in Escherichia coli and impact of overexpression of the soluble transhydrogenase UdhA. FEMS Microbiol Lett. 2001;204:247-52.

24. Meier S, Jensen PR, Duus J $\varnothing$. Direct observation of metabolic differences in living Escherichia coli strains K-12 and BL21. ChemBioChem. 2012;13:308-10

25. Hua Q, Yang C, Baba T, Mori H, Shimizu K. Responses of the central metabolism in Escherichia coli to phosphoglucose isomerase and glucose6-phosphate dehydrogenase knockouts. J Bacteriol. 2003;185:7053-67.

26. Chemler JA, Fowler ZL, McHugh KP, Koffas MA. Improving NADPH availability for natural product biosynthesis in Escherichia coli by metabolic engineering. Metab Eng. 2010;12:96-104.

27. Canonaco F, Hess TA, Heri S, Wang T, Szyperski T, Sauer U. Metabolic flux response to phosphoglucose isomerase knock-out in Escherichia coli and impact of overexpression of the soluble transhydrogenase UdhA. FEMS Microbiol Lett. 2001;204:247-52.

28. Flores S, de Anda-Herrera R, Gosset G, Bolívar FG. Growth-rate recovery of Escherichia coli cultures carrying a multicopy plasmid, by engineering of the pentose-phosphate pathway. Biotechnol Bioeng. 2004;87:485-94.

29. Walsh K, Koshland DE Jr. Characterization of rate-controlling steps in vivo by use of an adjustable expression vector. Proc Natl Acad Sci USA. 1985;82:3577-81.
30. Ow DSW, Nissom PM, Philp R, Oh SKW, Yap GS. Global transcriptional analysis of metabolic burden due to plasmid maintenance in Escherichia coli DH5a during batch fermentation. Enzyme Microb Technol. 2006;39:391-8.

31. Neubauer P, Lin HY, Mathiszik B. Metabolic load of recombinant protein production: inhibition of cellular capacities for glucose uptake and respiration after induction of a heterologous gene in Escherichia coli. Biotechnol Bioeng. 2003;83:5364.

32. Zhuang ZY, Li SY. Rubisco-based engineered Escherichia coli for in situ carbon dioxide recycling. Bioresour Technol. 2013;150:79-88.

33. Miller JH. Experiments in molecular genetics. New York: Cold Spring Harbor; 1972.

34. Saini M, Chiang CJ, Li SY, Chao YP. Production of biobutanol from cellulose hydrolysate by the Escherichia coli coculture system. FEMS Microbiol Lett. 2016. doi:10.1093/femsle/fnw008.

35. Chiang CJ, Chen PT, Chao YP. Replicon-free and markerless methods for genomic insertion of DNAs in phage attachment sites and controlled expression of chromosomal genes in Escherichia coli. Biotechnol Bioeng. 2008;101:985-95.

36. Saini M, Wang ZW, Chiang CJ, Chao YP. Metabolic engineering of Escherichia coli for production of butyric acid. J Agri Food Chem. 2014;62:4342-8

37. Snoep JL, Arfman N, Yomano LP, Westerhoff HV, Conway T, Ingram LO. Control of glycolytic flux in Zymomonas mobilis by glucose-6-phosphate dehydrogenase activity. Biotechnol Bioeng. 1996;15:191-7.

38. Sinha A, Maitra PK. Induction of specific enzymes of the oxidative pentose pathway by glucono-delta-lactone in Saccharomyces cerevisiae. J Gen Microbiol. 1992;138:1865-73.

\section{Submit your next manuscript to BioMed Central and we will help you at every step:}

- We accept pre-submission inquiries

- Our selector tool helps you to find the most relevant journal

- We provide round the clock customer support

- Convenient online submission

- Thorough peer review

- Inclusion in PubMed and all major indexing services

- Maximum visibility for your research

Submit your manuscript at www.biomedcentral.com/submit 